\title{
Acute Myeloid Leukemia in Adults
}

\author{
Jurjen Versluis, Jan J. Cornelissen, \\ Charles Craddock, Miguel Á. Sanz, \\ Jonathan Canaani, and Arnon Nagler
}

\subsection{AML in CR1}

Jurjen Versluis, Jan J. Cornelissen

\subsubsection{Definition, Subtypes}

AML is a malignancy of hematopoietic immature precursors (myeloblasts) that accumulate in the $\mathrm{BM}$ at the expense of their normal counterparts. AML is diagnosed by cytomorphology if more than $20 \%$ myeloblasts are present in the BM or PB. In addition, immunophenotyping and cytogenetic and molecular characterization are used to identify a number of AML subtypes.

J. Versluis · J. J. Cornelissen

Department of Hematology, Erasmus Medical Center

Cancer Institute, Rotterdam, The Netherlands

C. Craddock

Centre for Clinical Haematology, Queen Elizabeth Hospital, Birmingham, UK

M. Á. Sanz

Department of Hematology, University Hospital La

$\mathrm{Fe}$, University of Valencia, Valencia, Spain

J. Canaani · A. Nagler $(\bowtie)$

Hematology and BMT Division, Chaim Sheba

Medical Center, Tel-Hashomer, Israel

e-mail: Arnon.Nagler@sheba.health.gov.il
The WHO defines seven major subtypes of AML based largely on genetic, morphological, and cytochemical characteristics of the disease, whereas the most recent update (2016) included a new category termed "myeloid neoplasms with germ line predisposition" (Arber et al. 2016). In addition, risk classification is increasingly done according to the latest European LeukemiaNet (ELN) recommendations, whereby three risk groups are distinguished, including favorable risk, intermediate risk, and adverse risk, largely based on pretreatment cytogenetic abnormalities and aberrations in the NPM1, FLT3, CEBPA, RUNX1, ASXL1, and TP53 genes (Dohner et al. 2017).

\subsubsection{Clinical Presentation}

The median age at diagnosis is approximately 70 years, and the annual age-standardized incidence rate varies between 3 and 4 cases per 100,000. Patients with AML may present with symptoms such as fatigue and loss of appetite, whereas lymphadenopathy and hepatosplenomegaly may be found by physical examination. Analysis of blood work often reveals thrombocytopenia, anemia, and/or neutropenia. In some patients a serious bleeding diathesis can occur, particularly in the early phase of treatment, because the leukemic blasts are able to activate the coagulation cascade as well as cause hyperfibrinolysis. This particularly occurs in patients with acute promyelocytic leukemia (APL). 


\subsubsection{First-Line Treatment}

Achievement and maintenance of complete remission (CR) are crucial in younger AML patients aged below 60 years, but treatment may largely fail because of relapse from CR rather than primary resistance or treatment-related mortality (TRM).

With modern supportive care, TRM rates average $<5 \%$. For 30 years standard induction treatment (to produce $\mathrm{CR}$ ) has consisted of 7-10 days of the antimetabolite cytosine arabinoside (Ara-C) and 3 days of an anthracycline (i.e., daunorubicin or idarubicin). CR rates with standard induction estimate between 70 and 90\%. Favorable-risk patients may experience relatively good outcome with overall survival rates of approximately $60 \%$, whereas outcome for patients with intermediaterisk and adverse-risk AML remains unsatisfactory.

Following the introduction of targeted therapies in other malignancies including TKI in CML, studies to evaluate targeted treatment in AML were initiated 5-10 years ago, and a large randomized study in patients with FLT3 AML demonstrated a survival benefit for younger AML patients treated with the kinase inhibitor midostaurin in conjunction with intensive induction and consolidation chemotherapy (Stone et al. 2017). That study led to the approval of midostaurin, which has now become standard of care in AML patients with mutated FLT3. The ability of the pretreatment features such as incorporated in the ELN risk classification to predict outcome is important to direct treatment decisions; however probably more prognostically important than the pretreatment features is response to treatment (CR vs. lesser degrees of "response") and especially presence, in hematological remission, of "minimal residual disease" (MRD) as assessed by flow cytometry or molecular testing in patients with abnormalities such as mutated NPM1.

Allo-HSCT clearly reduces relapse rates but is associated with TRM (see sect. 69.1.4). Patients who do not qualify for HSCT are usually offered intensive consolidation chemotherapy based on high-dose cytarabine (HDAC). The dose of Ara-C has been a subject of study and intense debate questioning the application of dosages exceeding $2 \mathrm{~g} / \mathrm{m}^{2}$, which is now generally considered the upper dose (Lowenberg 2013). Induction chemotherapy in younger patients may include the $3+$ 7 scheme, whereas older patients may not tolerate intensive induction therapy and therefore are considered for non-intensive induction. However, it should be noted that also patients above the age of 60 years without comorbidities and no organ dysfunctions may be candidates for intensive therapy, which has been demonstrated to result in superior outcome (Lowenberg et al. 2009). Nevertheless, with a median age of approximately 70 years, most older patients are offered non-intensive therapy. With regard to non-intensive therapy, low-dose cytarabine (LDAC) is generally preferred over best supportive care (BSC) (Burnett et al. 2007). More recently, demethylation agents were compared with LDAC or BSC in older patients with AML and/or MDS, and both azacitidine (AZA) and decitabine showed a modest survival advantage. Although these agents resulted in a small improvement in overall survival, they may be preferred over LDAC or BSC in patients who cannot tolerate intensive induction therapy. At present the demethylating agents are predominantly used in those patients with myelodysplastic features.

\subsubsection{HSCT and AML Risk Categories}

\subsubsection{ELN Risk Categories}

Previously, conventional cytogenetics and mutations of NPM1, FLT3-ITD, and CEBPA were included in the ELN 2010 risk classification of AML patients (Dohner et al. 2010). The current ELN 2017 risk classification has added mutations in three genes including RUNX1, ASXL1, and TP53 (Table 69.1) (Dohner et al. 2017). Similar to the previous risk classification, the ELN 2017 AML risk classification is advocated to be used for risk-stratifying AML and to a risk-adapted treatment approach of patients with AML. Such a riskadapted treatment approach of patients with AML depends on the risk of relapse of the underlying AML but also on the risk of TRM associated with the applied post-remission treatment. The application of MRD, detected by either multiparametric 
Table 69.1 Risk-adapted post-remission treatment for patients with AML in first $\mathrm{CR}^{\mathrm{a}}$

\begin{tabular}{|c|c|c|}
\hline AML risk classification $^{\mathrm{b}}$ & MRD status ${ }^{\mathrm{c}}$ & $\begin{array}{l}\text { Preferred post-remission } \\
\text { treatment }\end{array}$ \\
\hline $\begin{array}{l}\text { Favorable } \\
\mathrm{t}(8 ; 21)(\mathrm{q} 22 ; \mathrm{q} 22.1) ; R U N X 1-R U N X 1 T 1 \\
\text { inv(16)(p13.1q22) or } \mathrm{t}(16 ; 16)(\mathrm{p} 13.1 ; \mathrm{q} 22) ; C B F B-M Y H 11 \\
\text { Mutated NPM1 without } F L T 3-I T D \text { or with FLT3-ITD low } \\
\text { Biallelic mutated CEBPA }\end{array}$ & $\begin{array}{l}\text { Negative } \\
\text { Positive }\end{array}$ & $\begin{array}{l}\text { Chemotherapy/auto-HSCT } \\
\text { Allo-HSCT }{ }^{\text {d }} \text {, (unless excessive } \\
\text { TRM can be predicted) }\end{array}$ \\
\hline $\begin{array}{l}\text { Intermediate } \\
\text { Mutated } N P M 1 \text { and } F L T 3-I_{T D}^{\text {high }} \\
\text { Wild-type } N P M 1 \text { without } F L T 3-\text { ITD or with } F L T 3-\text { ITD }^{\text {low }} \\
\text { (without adverse risk genetic lesions) } \\
\text { t }(9 ; 11)(\mathrm{p} 21.3 ; \mathrm{q} 23.3) ; M L L T 3-K M T 2 A \\
\text { Cytogenetic abnormalities not classified as favorable or adverse }\end{array}$ & Positive & $\begin{array}{l}\text { Allo-HSCT }^{\mathrm{d}} \\
\text { (if acceptable risk of TRM; } \\
\text { alternative, chemo/auto-HSCT) } \\
\text { Allo-HSCT }\end{array}$ \\
\hline 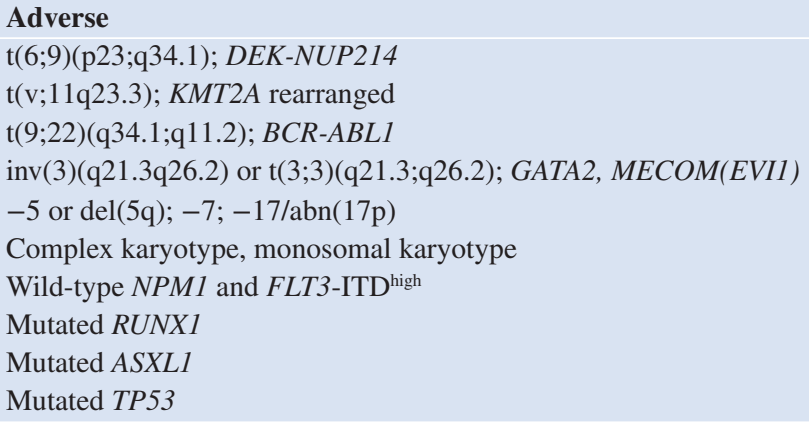 & $\begin{array}{l}\text { Negative } \\
\text { Positive }\end{array}$ & $\begin{array}{l}\text { Allo-HSCT } \\
\text { Allo-HSCT }^{\mathrm{e}}\end{array}$ \\
\hline $\begin{array}{l}\text { Adapted from Cornelissen et al. 2012a, b), Table } 4 \\
\text { Adapted from Dohner et al. (2017), Table } 5 \\
\text { Detected with multiparametric flow cytometry or with for qPCR sp } \\
\text { Allo-HSCT using HLA-identical sibling or 10/10 MUD donors }\end{array}$ & fic markers & \\
\hline
\end{tabular}

flow cytometry or quantitative PCR for specific molecular markers, may further improve AML risk classifications. MRD may be detected at time points early after induction treatment to assess the remission status of the AML but also after PRT to detect imminent relapse. Consequently, MRD negativity was introduced as an endpoint in patients with a hematological CR (Dohner et al. 2017).

\subsubsection{Transplant Risk Categories}

The risk-adapted approach of patients with AML in first CR should also include the assessment of TRM for each individual patient. TRM may be attributed to GVHD, infectious complications, organ toxicity, and other causes (Gooley et al. 2010). A number of parameters may relate to allo-HSCT-related TRM, including the procedure (e.g., conditioning regimen, application of TCD), donor characteristics (e.g., HLA-matching), and recipient features (e.g., age and comorbidity). The risk of mortality may be quantified by composite risk scores, which have been established to predict for TRM and overall outcome.

Two generally approved transplant risks were developed and validated, including the EBMT risk score (Gratwohl et al. 1998) and the hematopoietic cell transplantation-comorbidity index (HCT-CI) (Sorror et al. 2005). The EBMT risk score is based on patient and transplantation characteristics, which was developed in CML patients and subsequently validated in other patient groups including AML (Gratwohl et al. 2009). The HCT-CI originated from the Charlson comorbidity index and consists of 17 comorbidities which contribute to a cumulative score (Sorror et al. 2005). The HCT-CI was extensively validated and has been continuously being refined including age, disease status, or biomarkers 
(Sorror et al. 2007, 2014). Other groups have also developed predictive models for TRM modifying the weights of the EBMT risk score and the HCT-CI (Barba et al. 2010), whereas others combined transplant-related parameters and patient characteristics (Parimon et al. 2006; Barba et al. 2014; Shouval et al. 2015).

A more sophisticated, machine-based learning model was developed by the EBMT-acute leukemia working party (ALWP) based on 10 variables, which resulted in an alternating decision tree model highly predicting for mortality at 100 days and at 2 years (Shouval et al. 2015).

However, with the introduction of RIC, alloHSCT is increasingly being applied as postremission treatment for older or less fit patients with comorbidities. Several groups have reported less predictive power of the EBMT-score and the HCT-CI in these subgroups of patients as a number of comorbidities are less strongly associated with mortality after RIC than after MAC (Gratwohl et al. 2009; Barba et al. 2010; Barba et al. 2014). The EBMT-ALWP has developed an integrated score based on the EBMT risk score and the HCT-CI with increased predictive power in the setting of RIC allo-HSCT (Versluis et al. 2015). The lack of predictive power of the established risk scores and the development of a refined and dedicated model emphasize that prediction of TRM requires a continued reassessment of risk scores in specific patient groups.

\subsubsection{HSCT in First-Line AML Treatment: A Risk-Adapted Approach}

AML risk classifications are being used for tailoring patients' optimal post-remission treatment, which may include allo-HSCT, auto-HSCT, and continued chemotherapy. Allo-HSCT is the most optimal post-remission treatment for the prevention of relapse due to a potent GVL effect, which has been demonstrated to be exerted irrespective of underlying AML cytogenetic subcategories and MRD status (Cornelissen et al. 2012b; Versluis et al. 2017a). However, absolute estimates of relapse incidence differ and may reflect molecular or cytogenetic differences resulting in resistance of the AML. Although the GVL effect of alloHSCT is unequivocally present in patients with AML in first CR, concurrent TRM may compromise overall outcome, especially in AML patients with a relatively low incidence of relapse. Thus, a risk-adapted approach of post-remission treatment for patients with AML in first CR should include an assessment of the TRM risk profile in addition to leukemia characteristics and MRD (Cornelissen et al. 2012a; Cornelissen and Blaise 2016). Table 69.1 summarizes a risk-adapted approach based on the latest ELN AML risk classification, MRD status, and the risk for TRM. The risk for TRM should be preferably assessed with dedicated scores for specific subgroups of patients. Patients with MRD are considered high-risk for relapse and preferably receive an allo-HSCT in first CR, unless excessive NRM may be predicted.

Allo-HSCT is generally not being indicated in patients with a favorable AML risk profile; for those patients auto-HSCT or continued chemotherapy may be preferred (Dohner et al. 2017; Cornelissen et al. 2012a; Cornelissen and Blaise 2016). However, favorable-risk patients with persistent MRD may receive an allo-HSCT, especially those patients with a low risk for TRM.

Results of allo-HSCT compared with autoHSCT or chemotherapy have yielded contradicting results in intermediate-risk patients, especially taking molecular markers into account (Koreth et al. 2009; Schlenk et al. 2008; Rollig et al. 2015; Stelljes et al. 2014; Versluis et al. 2017b). Assessment of the MRD status is strongly advocated for patients with an intermediate-risk AML. Allo-HSCT may be applied in patients with intermediate-risk AML with MRD after induction chemotherapy, except for patients with a high risk for TRM. Allo-HSCT is also preferred for patients with intermediate-risk MRD-negative AML, but auto-HSCT or chemotherapy may be considered when the predicted risk for TRM is high.

Adverse-risk patients with MRD should be transplanted with an allografted as soon as a hematological $\mathrm{CR}$ is obtained. Adverse-risk patients without MRD still have a significant risk of relapse and may also receive an allo-HSCT, although patients with a very high risk for TRM may alternatively receive autologous HSCT or a third cycle of chemotherapy. 


\subsection{Allo-HSCT in Advanced AML}

Charles Craddock

\subsubsection{Introduction}

Allo-HSCT plays an increasingly important role in the management of AML in adults (Cornelissen et al. 2012a). The advent of RIC regimens coupled with increased donor availability has dramatically increased the number of patients in whom allo-HSCT can be contemplated.

At the same molecular characterization at diagnosis coupled with measurable MRD quantitation after induction, chemotherapy has considerably improved our ability to predict relapse risk in patients treated with intensive chemotherapy (IC) alone permitting accurate identification of allo-mandatory patients.

As a result, allo-HSCT in patients with AML in CR1 is an increasingly important personalized component of the treatment algorithm. At the same time, transplantation is also emerging as an important, potentially curative treatment modality in patients with advanced AML.

The increasingly important role of allo-HSCT in the management of AML mandates the development of novel strategies with the potential to improve transplant outcome. Although the last three decades has witnessed a substantial reduction in TRM, the risk of disease relapse post transplant remains stubbornly high and now represents the major cause of treatment failure in patients allografted for AML. There is consequently an urgent requirement to develop novel strategies with the potential to reduce the risk of disease recurrence.

\subsubsection{The Role of Allo-HSCT in the Management of AML Beyond CR1}

While a small proportion of patients with AML in CR2 achieve long-term survival if treated with salvage chemotherapy alone, compelling data identify allo-HSCT as the preferred curative option (Gale et al. 1996). Long-term survival rates in the region of 30-50\% have been reported after both transplantation from either a matched sibling or MUD (Tauro et al. 2005). Encouraging results are also reported in patients transplanted using CBU with a good total nucleated cell dose and, more recently, haploidentical donors. In patients who have achieved a morphological CR2, it is wise to proceed immediately to transplant, providing the patient is fit and a donor has been identified and there is no evidence supporting further courses of chemotherapy prior to transplantation.

While a rigorous comparison of MAC and RIC regimens has not been performed in fit patients under the age of 50, a MAC regimen should probably be preferred. Retrospective studies have demonstrated that auto-HSCT can achieve comparable results to those observed with an allogeneic donor in adults with AML associated with a CBF abnormality, and this can represent an important treatment option in patients with comorbidities or in the absence of a well-matched donor (Gorin et al. 2008), particularly if both the patient and stem cell graft are MRD negative.

Ten to forty percent of adults with newly diagnosed AML fail to achieve a morphological CR after two courses of induction chemotherapy (Ferguson et al. 2016). Factors determining refractoriness to induction therapy include patient age and the presence of an adverse risk karyotype. Although recognized as one of the most important causes of treatment failure, it is perhaps surprising that there is no consensus definition of primary refractory AML (PREF AML). While the International Working Group (IWG) and the European LeukemiaNet (ELN) define resistant disease as persistent leukemic blasts following one course of induction chemotherapy in either the PB or the $\mathrm{BM}$ in a patient alive 7 days or more following treatment (Cheson et al. 2003; Dohner et al. 2010), most transplant studies instead have classified PREF AML as a failure to achieve a morphological CR after two induction courses.

The UK NCRI group recently studied more than 8000 patients with the aim of more precisely defining a measure of chemo-refractoriness. Patients with greater than $15 \%$ residual blasts or less than a $50 \%$ proportional reduction in blast count after the first course of induction chemotherapy were observed to have similar outcomes to patients who fail to achieve a morphological CR 
after two courses. Such patients were observed to possess genuinely chemo-refractory disease with long-term survival rates $<10 \%$ if treated with chemotherapy alone (Ferguson et al. 2016). In contrast, patients who fulfilled either definition of refractory disease achieved long-term survival rates in the region of $25-30 \%$ after allo-HSCT.

Evidence that allo-HSCT can deliver longterm survival in a significant proportion of patients with PREF AML has been accumulating over the last decade and represents an important advance in management of this sizeable patient population for whom no other effective therapy exists (Craddock et al. 2011; Todisco et al. 2017; Brissot et al. 2017). Nonetheless outcomes in patients allografted for PREF AML remain unsatisfactory, and both TRM and disease relapse continue to represent significant barriers to long-term survival. There is also a lack of clarity concerning which patients with PREF AML are the most likely to benefit from transplantation. Outcome is clearly superior in patients who proceed swiftly to transplant after no more than two courses of IC, and relapse appears to be lower in those with a lower burden of disease at the time of transplantation. Importantly the impact of presentation karyotype and genotype on remains undetermined although some studies, perhaps unsurprisingly, identify worse outcome in patients with a complex karyotype. It is therefore important that further studies examining the impact of presentation karyotype, mutational status, and pre-transplant disease load on outcome after allo-HSCT are prioritized. What is incontrovertible however is that adults with high-risk AML should undergo an urgent search for sibling and URD at presentation so that transplant can be swiftly scheduled if the patients are refractory to chemotherapy.

The optimal conditioning regimen in patients with PREF AML remains a matter of conjecture. While MAC regimens should be preferred in fit patients under the age of 50, encouraging results have also been reported using the sequential FLAMSA regimen which incorporates additional tumor debulking, using Ara-C and amsacrine, prior to a FLU-based RIC regimen (Schmid et al. 2006). Importantly this schedule also incorporates early administration of DLI, at day +120 , in patients with no evidence of active GVHD.

\subsubsection{Strategies to Prevent Disease Relapse in Patients Allografted for AML}

Disease relapse remains the major cause of treatment failure in patients allografted for AML (Cornelissen et al. 2012b). Despite substantial progress in reducing the toxicity of allo-HSCT, the risk of disease recurrence remains stubbornly high, and novel strategies with the potential to reduce the risk of disease recurrence are urgently required (Craddock et al. 2018). Key to the development of effective new interventions is an understanding of both the clinical factors determining disease relapse and an improved understanding of the biology of disease recurrence (Ossenkoppele et al. 2016). In addition to the impact of presentation karyotype, next-generation sequencing (NGS) studies have identified molecular determinants of disease relapse post transplant (Lindsley et al. 2017). Retrospective studies have also demonstrated that pre-transplant MRD is an important predictor of disease relapse after allo-HSCT, although confirmation of these data in prospective trials is still lacking (Walter et al. 2011).

The risk of disease relapse also appears to be impacted by the conditioning regimen, and retrospective studies consistently demonstrate an increased risk of recurrence in patients transplanted using a RIC regimen although recent prospective randomized trials have yielded conflicting data (Fasslrinner et al. 2018; Kroger et al. 2017; Scott et al. 2017). Finally, the intensity of post transplant IS is also a critical factor influencing relapse risk consistent with the exertion of a potent GVL effect in patients allografted for AML (Bacigalupo et al. 1991; Craddock et al. 2010).

A number of novel approaches toward reducing post transplant relapse are currently undergoing evaluation. Firstly, quantitation of pre-transplant MRD status, using immunophenotypic or molecular methodologies, can identify patients with a higher risk of relapse and has resulted in exploration of approaches which reduce the pre-transplant MRD status as a means of improving transplant outcome. Pivotal to the implementation of such strategies are reproducible and accurate measurements of pre-transplant MRD status, and of note novel NGS technologies 
with improved sensitivity are emerging (JongenLavrencic et al. 2018). Secondly, identification of the optimal conditioning regimen remains key to optimizing transplant outcome. One of the most important considerations in interpretation of comparisons of MAC and RIC regimens will be whether pre-transplant MRD influences patient outcome in a regimen-dependent manner.

Finally, there is increasing interest in the elective administration of pharmacological agents or cellular therapies post transplant. A number of agents are currently under evaluation as post transplant maintenance strategies including targeted therapies such as Flt3 inhibitors or agents with a broader antileukemic activity including demethylating agents such as AZA or checkpoint inhibitors (Craddock et al. 2016; Soiffer et al. 2018). In the future it is likely that the choice of maintenance strategies will be informed by a greater understanding of the biology of disease recurrence. In this context it is of interest that a significant number of patients who relapse post-allograft demonstrate loss or acquisition of candidate driver mutations at the time of relapse (Quek et al. 2016).

\subsection{Practical Issues in Allo-HSCT for AML}

Jonathan Canaani, Arnon Nagler

\subsubsection{Stem Cell Source} (See Also Chaps. 14 and 15)

One of the fundamental issues in the initial decision-making for transplantation physicians is the optimal source for procuring the stem cells for transplant. Whereas the initial methodology for donor stem cell procurement involved direct $\mathrm{BM}$ harvesting, the introduction of PBSC mobilization more than two decades ago into routine clinical practice has shifted the field toward the latter approach. Indeed, it has been estimated that PBSC is used in more than $75 \%$ of allo-HSCT (per National Marrow Donor Program data; http://www.marrow.org).

Whether PBSC are preferable to BM harvesting as the stem cell source for patients with
AML is still an open question; however there are several noteworthy facts which need to be mentioned. In patients receiving grafts from MSD, early publications suggested superior engraftment rates in PBSC concomitant to an increased risk of acute and chronic GVHD in some of the studies (Couban et al. 2002). A phase 3 study was conducted by the BMT CTN randomized patients with various myeloid malignancies (including 261 AML patients) to receive PBSC versus BM harvested cells from MUD (Anasetti et al. 2012). The results of this pivotal study revealed comparable rates of survival and relapse between both groups with increased rates of graft failure in the bone marrow group counterbalanced by an increased likelihood of chronic GVHD in the PBSC group. Two analyses from the EBMT and the CIBMTR in the RIC setting also confirmed the absence of a survival difference for either approach (Nagler et al. 2012; Eapen et al. 2015). Thus, it seems reasonable to conclude that at present both stem cell sources are acceptable options to use in AML patients.

\subsubsection{Best Donor (See Also Chap. 12)}

AML patients referred to transplant are currently candidates for several potential donor sources including HLA MSD, HLA MUD, UCB grafts, and haploidentical donors. Indeed, the rapid evolution of the field of HSCT is possibly best exemplified by the potential donor pool which has expanded from the initial requirement for an HLA-matched sibling to include also MUD, UCB grafts, and more recently also use of haploidentical donors. While MSD and MUD remain the preferred donor source in most clinical settings (Schlenk et al. 2010), the accumulating experience with UCB and haplo donors provides a much-needed donor resource for those patients lacking suitable MSD or MUD donors, a need especially evident in minority populations.

The original pediatric experience with UCB has been successfully translated into adult transplantation protocols both in the RIC and MAC settings (Oran et al. 2011). Published data from a joint CIBMTR/EBMT retrospective study comparing UCB and MUD transplants in over 1500 
acute leukemia patients suggested equivalent LFS rates in both groups (Eapen et al. 2010). Of note, UCB patients in this study had higher TRM rates but a lower incidence of acute and chronic GVHD. Interestingly, an analysis in high-risk AML patients who underwent either RIC UCB transplant or RIC MSD/MUD revealed that the incidence of relapse was more than doubled in the UCB group (Devillier et al. 2014), while a CIBMTR/Eurocord retrospective study of patients over the age of 50 showed that UCB transplant is feasible in this age group albeit at the price of an increased rate of TRM and lower LFS rate (Weisdorf et al. 2014). Whether a two-unit UCB transplant is superior to a one-unit UCB transplant is not entirely clear at this point; however a randomized study conducted in pediatric and adolescent patients indicates similar survival and relapse rates between both groups in addition to improved rates of grade III/IV acute and extensive chronic GVHD in those patients receiving a single unit of UCB (Wagner Jr. et al. 2014).

The inherent benefit in using haplo donors is the near-universal availability of several potential donors which could be either siblings, parents, or children dependent on the patient's age. The initial experience with this approach was limited by a substantial component of TRM due to the slow kinetics of immune reconstitution resulting in infectious complications as well as graft rejection (Ciurea et al. 2015). A significant breakthrough was realized with the advent of novel IS modulation approaches such as PT-CY-based (Robinson et al. 2016) and ATG-based protocols (Chang et al. 2014), which via selective in vivo TCD have achieved acceptable rates of engraftment. An evolving body of literature suggests comparable outcomes between haplo transplantation and transplantation from partially HLA MMUD and possibly MUD and MSD as well (Bashey et al. 2013). In conclusion, when available, MSD and 10/10 HLA MUD remain the first choice for donors. For patients lacking MSD/MUD, both UCB and haplo donors are reasonable alternative donor sources.

\subsubsection{Conditioning (See Also Chap. 13)}

The ideal conditioning regimen for patients with AML is a yet unsettled question in the field of transplantation. Yet, the pivotal point to initially consider when deciding on a specific conditioning regimen is whether the patient would be eligible to receive MAC or rather RIC. For younger (less than 45 years of age for most MAC candidates) and fit patients, MAC is the preferred choice given its superior antileukemia activity shown in previous studies (Martino et al. 2013) and especially in light of the recent data presented by the BMT CTN 0901 trialists underscoring the marked relapse-free survival advantage experienced by MAC patients compared to RIC patients (67\% vs. 47\%) (Scott et al. 2017).

From a toxicity standpoint, older patients derive the most benefit from RIC resulting in more favorable NRM and TRM rates. Notably, the incidence of GVHD, late infectious complications, and CMV reactivation is comparable between MAC and RIC, while the incidence of acute transplant complications (i.e., SOS/VOD, mucositis, IPS, and hemorrhagic cystitis) is more common in MAC and provides the advantage NRM for RIC (Sengsayadeth et al. 2015).

\subsubsection{Graft Versus Host Disease Prophylaxis (See Also Chap. 25)}

Up to $70 \%$ of transplanted patients will experience acute GVHD to some extent, and these patients are at a significant risk of morbidity and mortality resulting from this severe inflammatory reaction. Thus, from a therapeutic standpoint, prophylaxis of acute GVHD is one of the crucial intervention points during the process of alloHSCT. In current practice standard prophylaxis regimens for acute GVHD comprise the dual use of a CNI, namely, CSA or TAC, added to MTX or MMF for the first 180 days following transplantation (Ruutu et al. 2014). Published data from studies conducted two decades ago suggested that TAC/MTX was superior to CSA/MTX in terms of acute GHVD, however this did not translate into a survival advantage, and in fact the latter regimen may be more commonly used among transplant centers (Nash et al. 2000). MMF, an inosine monophosphate dehydrogenase inhibitor, is not often used in the MAC setting, and currently its role is mostly limited to CBT and 
non-MAC-HSCT. SIR (rapamycin) was evaluated in several phase I/II with non-heterogeneous patient cohorts which limited interpretation of its efficacy; however a phase III BMT CTN study did not show an improvement in the incidence of GVHD over MTX (Cutler et al. 2014).

Whereas the abovementioned therapeutic modalities are quite adept at prevention of acute GVHD, preventing chronic GVHD is still a major challenge with these agents, and thus more specialized strategies to mitigate GVHD have been attempted including TCD accomplished using either ex vivo (via positive selection of CD34positive cells or through negative depletion of specific T cell subsets) (Saad and Lamb 2017) or in vivo methodologies (by use of TCD drugs such as ALEM or ATG). Recent publications from several phase III studies clearly demonstrate using ATG was beneficial for patients with acute leukemia as well as other hematological malignancies (Kroger et al. 2016). Ex vivo TCD is a promising approach and has been shown to be effective for GVHD prophylaxis in smaller trials (Pasquini et al. 2012), although wider application of this methodology will require further data.

\subsection{Acute Promyelocytic Leukemia}

Miguel Á. Sanz

\subsubsection{Concept and Incidence}

APL is a subtype of AML with peculiar clinical and morphological characteristics that presents a specific genetic alteration, the $t(15 ; 17)$, with its corresponding molecular counterpart, the rearrangement $P M L-R A R A$, which confer a particular sensitivity to all-trans retinoic acid (ATRA) and arsenic trioxide (ATO). It also highlights the presence of a hemorrhagic diathesis associated with a peculiar coagulopathy, which causes a high incidence of hemorrhagic complications at presentation and early during the induction treatment.

APL accounts for 10-15\% of AML.

\subsubsection{Diagnosis}

\subsubsection{Morphology, Immunophenotyping, and Other Features}

\begin{tabular}{|c|c|}
\hline $\begin{array}{l}\text { M3 typical } \\
\text { (hypergranular) }\end{array}$ & $\begin{array}{l}\text { M3 variant } \\
\text { (microgranular) }\end{array}$ \\
\hline \multicolumn{2}{|l|}{ Morphology } \\
\hline $\begin{array}{l}\text { - Cytoplasm with dense } \\
\text { granulation. Frequent } \\
\text { Auer rods } \\
\text { - Reniform or bilobed } \\
\text { nucleus }\end{array}$ & $\begin{array}{l}\text { - Cytoplasm with fine } \\
\text { granulation or } \\
\text { hypogranular. Less } \\
\text { frequent Auer rods } \\
\text { - Reniform nucleus, bi- or } \\
\text { multilobed }\end{array}$ \\
\hline \multicolumn{2}{|l|}{ Immunophenotyping } \\
\hline $\begin{array}{l}\mathrm{HLA}^{-\mathrm{DR}}{ }^{-} / \mathrm{CD}^{3} 4^{-} / \mathrm{CD}^{2} 3^{+\mathrm{a}} / \\
\mathrm{CD} 13^{+\mathrm{b}} / \mathrm{CD} 15^{-/+}\end{array}$ & $\begin{array}{l}\mathrm{HLA}-\mathrm{DR}^{ \pm} / \mathrm{CD} 34^{ \pm} / \mathrm{CD} 33^{+\mathrm{a}} / \\
\mathrm{CD}^{+} / \mathrm{CD} 2^{ \pm} / \mathrm{CD} 13^{+\mathrm{b}} / \\
\mathrm{CD}^{2} 6^{ \pm}\end{array}$ \\
\hline \multicolumn{2}{|l|}{ Other associated features } \\
\hline $\begin{array}{l}\text { - Most frequently, low } \\
\text { WBC counts } \\
\text { - Less frequently, BCR3 } \\
\text { isoform }\end{array}$ & $\begin{array}{l}\text { - Most frequently, high } \\
\text { WBC counts } \\
\text { - Most frequently, BCR3 } \\
\text { isoform }\end{array}$ \\
\hline
\end{tabular}

${ }^{a}$ Intense and homogeneous expression

${ }^{\mathrm{b}}$ Heterogeneous expression

\subsubsection{Genetic Diagnosis}

\begin{tabular}{|c|c|}
\hline \multicolumn{2}{|c|}{ Conventional cytogenetics $t(15 ; 17)(q 22 ; q 21)$} \\
\hline \multirow[t]{2}{*}{ Pros } & - Very specific \\
\hline & $\begin{array}{l}\text { - Detects additional anomalies in } 30 \%(+8 \\
\text { the most frequent) }\end{array}$ \\
\hline \multirow[t]{2}{*}{ Cons } & - Low sensitivity $(80 \%)$ \\
\hline & $\begin{array}{l}\text { - Inadequate, bad metaphases or normal } \\
\text { karyotype (false negative) in } 20 \%\end{array}$ \\
\hline \multicolumn{2}{|c|}{ FISH $P M L-R A R A$} \\
\hline Pros & - Very specific and rapid \\
\hline Cons & $\begin{array}{l}\text { - Not very sensitive and does not provide } \\
\text { information about the isoform }\end{array}$ \\
\hline \multicolumn{2}{|c|}{$R T-P C R$} \\
\hline \multirow[t]{2}{*}{ Pros } & - Very specific, rapid, and sensitive \\
\hline & $\begin{array}{l}\text { - Identifies the isoform, which allows MRD } \\
\text { monitoring }\end{array}$ \\
\hline Cons & - Occasional artifacts and contaminations \\
\hline \multicolumn{2}{|c|}{ Immunostaining with anti-PML antibody $(P G-M 3)$} \\
\hline \multirow[t]{2}{*}{ Pros } & - Very specific, rapid, and cheap \\
\hline & $\begin{array}{l}\text { - Characteristic microspeckled pattern by } \\
\text { indirect immunofluorescence }\end{array}$ \\
\hline Cons & $\begin{array}{l}\text { - Does not provide information about the } \\
\text { isoform }\end{array}$ \\
\hline
\end{tabular}




\subsubsection{Other Rearrangements of the RARA Gene on Chromosome 17}

Chromosomal abnormality

- $\mathrm{t}(11 ; 17)(\mathrm{q} 23 ; \mathrm{q} 21)$

- $\mathrm{t}(17 ; 17)(\mathrm{q} 21 ; \mathrm{q} 21)$

- $\mathrm{t}(11 ; 17)(\mathrm{q} 23 ; \mathrm{q} 21)$

- $\mathrm{t}(5 ; 17)(\mathrm{q} 35 ; \mathrm{q} 21)$

- $\mathrm{t}(11 ; 17)(\mathrm{q} 13 ; \mathrm{q} 21)$

- $\mathrm{t}(17 ; 17)(\mathrm{q} 21 ; \mathrm{q} 24)$

- $\mathrm{t}(\mathrm{X} ; 17)(\mathrm{p} 11 ; \mathrm{q} 21$

- $\mathrm{t}(4 ; 17)(\mathrm{q} 12 ; \mathrm{q} 21)$

- $\mathrm{t}(2 ; 17)(\mathrm{q} 32 ; \mathrm{q} 21)$

- $\mathrm{t}(3 ; 17)$ (q26;q21)

- $\mathrm{t}(7 ; 17)(\mathrm{q} 11 ; \mathrm{q} 21)$

- $\mathrm{t}(1 ; 17)(\mathrm{q} 42 ; \mathrm{q} 21)$
$R A R A$ rearrangement

- $P L Z F / R A R A$ (poorly responsive to ATRA)

- STAT5b/RARA (poorly responsive to ATRA)

- $K M T 2 a / R A R A$ (ATRA sensitivity unknown)

- NPM/RARA (ATRA sensitivity unknown)

- NuMA1-RARA (ATRA sensitivity unknown)

- PRKARla/RARA (ATRA sensitive)

- bCor/RARA (ATRA sensitive in two cases)

- FIP1L1/RARA (ATRA sensitivity unknown)

- obFC2A/RARA (ATRA sensitive in one case)

- $T B L R 1 / R A R A$ (insensitive to ATRA)

- gTF2l/RARA (ATRA sensitive)

- IRF2BP2/RARA (ATRA sensitive)

\subsubsection{First-Line Treatment}

The European LeukemiaNet (ELN) recommendations in 2009 already recognized the promising results reported in several non-randomized studies using ATRA plus ATO, with or without minimal use of chemotherapy, but the standard of care was still considered the combination of ATRA plus anthracycline-based chemotherapy (Sanz et al. 2009). However recent findings have led to modify this recommendation.

The long-term results of a non-randomized study (Abaza et al. 2017) and two recently reported randomized clinical trials (Lo-Coco et al. 2013; Burnett et al. 2015), comparing the efficacy and safety of ATRA plus ATO versus the standard ATRA plus chemotherapy approach, strongly support the former combination as the new standard of care for patients with low-tointermediate-risk APL with WBC counts lower than $10 \times 10^{9} / \mathrm{L}$ at presentation. Nevertheless, in countries where chemotherapy is more affordable than ATO, the classical combination of ATRA and chemotherapy is still an acceptable option. For high-risk patients, however, there are two valid options, either ATRA plus chemotherapy or ATRA plus ATO with a certain amount of cytoreductive chemotherapy, at least during the induction phase.

HSCT is never indicated in patients in CR1, except for the small fraction of patients with persistent RQ-PCR positivity of PML-RARA after consolidation $(<1 \%)$, given the poor prognosis of this subset of patients. HSCT is also indicated in APL patients who relapse and achieve second or subsequent $\mathrm{CR}$.

\subsubsection{Salvage Therapy}

Apart from patients with MRD positivity at the end of consolidation (molecular persistence), there is a general agreement that patients with the more common molecular or hematological relapse later on require immediate additional treatment, including HSCT. Salvage treatment should be given to attempt to achieve molecular remission as a bridge to HSCT. Salvage treatment with ATRA plus ATO is recommended when ATRA plus chemotherapy has been previously used front-line, whereas ATRA plus chemotherapy would be the option when front-line therapy was ATRA plus ATO.

The use of gemtuzumab ozogamicin may also be considered in both situations, but always as a bridge to HSCT. Based on recent studies, (Yanada et al. 2013; Holter Chakrabarty et al. 2014; Lengfelder et al. 2015) auto-HSCT should be considered the first choice for eligible patients achieving second molecular remission. Patients unsuitable for HSCT and those with a very prolonged CR1 can be managed with some type of continuation therapy which would be chosen taking into account previous treatments and clinical condition.

Allo-HSCT should be reserved for patients with high risk of relapse and low risk of TRM but also as a second option, for those who relapse after an auto-HSCT. 


\subsubsection{Indications of HSCT}

HSCT is never indicated in patients in CR1, except for those patients who do not achieve molecular remission at the end of consolidation $(<1 \%)$. Indications of HSCT and other recommendations for patients in whom HSCT is indicated are summarized in Table 69.1.

\subsubsection{Main Series Reported on HSCT in APL}

There are no randomized trials to evaluate the efficacy and safety of the different modalities of HSCT in refractory/relapsed APL. The data come mostly from retrospective studies comparing historical cohorts from registries (Tables 69.2 and 69.3).

Table 69.2 Indications of HSCT in patients with APL

\begin{tabular}{|c|c|c|}
\hline & Auto-HSCT & Allo-HSCT \\
\hline Not indicated & CR1 in molecular remission & CR1 in molecular remission \\
\hline Indicated & $\geq \mathrm{CR} 2$, but in molecular remission & $\begin{array}{l}-\geq \mathrm{CR} 2 \text { with } P M L-R A R A(+) \text { after salvage } \\
\text { therapy } \\
-\geq \mathrm{CR} 2 \text { if an auto-HSCT has failed previously } \\
-\geq \mathrm{CR} 2 \text { in patients with high risk of relapse } \\
\text { and low risk of TRM }\end{array}$ \\
\hline $\begin{array}{l}\text { Salvage therapy as } \\
\text { a bridge to HSCT }\end{array}$ & $\begin{array}{l}\text { Attempt to achieve molecular remission } \\
\text { with ATRA plus ATO in patient who } \\
\text { relapsed after ATRA plus chemotherapy as } \\
\text { front-line therapy, whereas ATRA plus } \\
\text { chemotherapy is the option when patients } \\
\text { relapse after ATRA plus ATO }\end{array}$ & $\begin{array}{l}\text { Attempt to achieve molecular remission with } \\
\text { ATRA plus ATO in patient who relapsed after } \\
\text { ATRA plus chemotherapy as front-line therapy, } \\
\text { whereas ATRA plus chemotherapy is the option } \\
\text { when patients relapse after ATRA plus ATO }\end{array}$ \\
\hline $\begin{array}{l}\text { Conditioning } \\
\text { regimen }\end{array}$ & $\begin{array}{l}\text { Either for use in AML, preferably } \\
\text { containing HDAC (e.g., BEA } \\
\text { (Gondo et al. 1997): BU/VP/Ara-C) }\end{array}$ & Either for use in AML \\
\hline Cell source & Mobilized peripheral blood & Mobilized peripheral blood \\
\hline $\begin{array}{l}\text { Indication of CNS } \\
\text { prophylaxis }\end{array}$ & $\begin{array}{l}\text { ITT with MTX, hydrocortisone, and Ara-C, } \\
\text { especially in those who presented relapse } \\
\text { in CNS }\end{array}$ & $\begin{array}{l}\text { ITT with MTX, hydrocortisone, and Ara-C, } \\
\text { especially in those who presented relapse in CNS }\end{array}$ \\
\hline $\begin{array}{l}\text { Maintenance } \\
\text { therapy post-HSCT }\end{array}$ & $\begin{array}{l}\text { Not proven, but conceivable that } \\
\text { ATO + ATRA may be effective }\end{array}$ & $\begin{array}{l}\text { Not proven, but conceivable that ATO + ATRA } \\
\text { may be effective }\end{array}$ \\
\hline $\begin{array}{l}\text { Molecular } \\
\text { monitoring }\end{array}$ & $\begin{array}{l}\text { Recommended by RQ-PCR at least every } \\
3 \text { months for } 2-3 \text { years }\end{array}$ & $\begin{array}{l}\text { Recommended by RQ-PCR at least every } \\
3 \text { months for } 2-3 \text { years }\end{array}$ \\
\hline
\end{tabular}

Table 69.3 Main series reported on HSCT in APL

\begin{tabular}{|l|c|l|l|}
\hline $\begin{array}{l}\text { Group and reference } \\
\text { European APL Group } \\
\text { (Thomas et al. 2000) }\end{array}$ & Patients & $\begin{array}{l}\text { Type of study } \\
\text { - Retrospective } \\
\text { - Inclusion of patients from the } \\
\text { pre-ATRA era }\end{array}$ & $\begin{array}{l}\text { Main conclusions } \\
\text { - Higher morbidity and TRM with } \\
\text { allo-HSCT } \\
\text { - Short follow-up }\end{array}$ \\
\hline $\begin{array}{l}\text { EBMT } \\
\text { (Sanz et al. 2007) }\end{array}$ & 332 & $\begin{array}{l}\text { - Retrospective } \\
\text { - Only patients of the pre-ATRA era }\end{array}$ & $\begin{array}{l}\text { - Higher TRM but lower relapse rate } \\
\text { with allo-HSCT compared with } \\
\text { auto-HSCT }\end{array}$ \\
\hline $\begin{array}{l}\text { Japan Adult Leukemia } \\
\text { Study Group (Yanada } \\
\text { et al. 2013) }\end{array}$ & 35 & $\begin{array}{l}\text { - Prospective, phase II, multicenter } \\
\text { - Salvage therapy with ATO+Ida, } \\
\text { followed by ATO x 2, HDAC, and } \\
\text { auto-HSCT }\end{array}$ & $\begin{array}{l}\text { - Outstanding efficacy and feasibility of } \\
\text { the sequential treatment featuring ATO } \\
\text { and auto-HSCT for relapsed APL }\end{array}$ \\
\hline $\begin{array}{l}\text { IBMTR (Holter } \\
\text { Chakrabarty et al. 2014) }\end{array}$ & 294 & $\begin{array}{l}\text { - Retrospective } \\
\text { - Only patients of the pre-ATRA era }\end{array}$ & $\begin{array}{l}\text { - Auto-HSCT yields superior OS for } \\
\text { APL in CR2 }\end{array}$ \\
\hline $\begin{array}{l}\text { ELN registry } \\
\text { (Lengfelder et al. 2015) }\end{array}$ & 155 & $\begin{array}{l}\text { - Retrospective } \\
\text { - Salvage therapy with ATO } \\
\text { for induction and consolidation } \\
\text { followed by auto- or allo-HSCT }\end{array}$ & $\begin{array}{l}\text { - Good but similar results with allo- and } \\
\text { auto-HSCT }\end{array}$ \\
\hline
\end{tabular}




\section{References}

Abaza Y, Kantarjian H, Garcia-Manero G, et al. Longterm outcome of acute promyelocytic leukemia treated with all-trans-retinoic acid, arsenic trioxide, and gemtuzumab. Blood. 2017;129:1275-83.

Anasetti C, Logan BR, Lee SJ, et al. Peripheral-blood stem cells versus bone marrow from unrelated donors. N Engl J Med. 2012;367:1487-96.

Arber DA, Orazi A, Hasserjian R, et al. The 2016 revision to the World Health Organization classification of myeloid neoplasms and acute leukemia. Blood. 2016;127:2391-405.

Bacigalupo A, Van Lint MT, Occhini D, et al. Increased risk of leukemia relapse with high-dose cyclosporine A after allogeneic marrow transplantation for acute leukemia. Blood. 1991;77:1423-8.

Barba P, Pinana JL, Martino R, et al. Comparison of two pretransplant predictive models and a flexible HCT-CI using different cut off points to determine low-, intermediate-, and high-risk groups: the flexible HCT-CI Is the best predictor of NRM and OS in a population of patients undergoing alloRIC. Biol Blood Marrow Transplant. 2010;16: 413-20.

Barba P, Martino R, Perez-Simon JA, et al. Combination of the Hematopoietic Cell Transplantation Comorbidity Index and the European Group for Blood and Marrow Transplantation score allows a better stratification of high-risk patients undergoing reduced-toxicity allogeneic hematopoietic cell transplantation. Biol Blood Marrow Transplant. 2014;20:66-72.

Bashey A, Zhang X, Sizemore CA, et al. T-cell-replete HLA-haploidentical hematopoietic transplantation for hematologic malignancies using post-transplantation cyclophosphamide results in outcomes equivalent to those of contemporaneous HLA-matched related and unrelated donor transplantation. J Clin Oncol. 2013;31:1310-6.

Brissot E, Labopin M, Stelljes M, et al. Comparison of matched sibling donors versus unrelated donors in allogeneic stem cell transplantation for primary refractory acute myeloid leukemia: a study on behalf of the Acute Leukemia Working Party of the EBMT. J Hematol Oncol. 2017;10:130.

Burnett AK, Milligan D, Prentice AG, et al. A comparison of low-dose cytarabine and hydroxyurea with or without all-trans retinoic acid for acute myeloid leukemia and high-risk myelodysplastic syndrome in patients not considered fit for intensive treatment. Cancer. 2007;109:1114-24.

Burnett AK, Russell NH, Hills RK, et al. Arsenic trioxide and all-trans retinoic acid treatment for acute promyelocytic leukaemia in all risk groups (AML17): results of a randomised, controlled, phase 3 trial. Lancet Oncol. 2015;16:1295-305.

Chang YJ, Wang Y, Huang XJ. Haploidentical stem cell transplantation for the treatment of leukemia: current status. Expert Rev Hematol. 2014;7:635-47.
Cheson BD, Bennett JM, Kopecky KJ, et al. Revised recommendations of the International Working Group for diagnosis, standardization of response criteria, treatment outcomes, and reporting standards for therapeutic trials in acute myeloid leukemia. J Clin Oncol. 2003;21:4642-9.

Ciurea SO, Zhang MJ, BacigalupoAA, et al. Haploidentical transplant with posttransplant cyclophosphamide vs matched unrelated donor transplant for acute myeloid leukemia. Blood. 2015;126:1033-40.

Cornelissen JJ, Blaise D. Hematopoietic stem cell transplantation for patients with AML in first complete remission. Blood. 2016;127:62-70.

Cornelissen JJ, Gratwohl A, Schlenk RF, et al. The European LeukemiaNet AML Working Party consensus statement on allogeneic HSCT for patients with AML in remission: an integrated-risk adapted approach. Nat Rev Clin Oncol. 2012a;9:579-90.

Cornelissen JJ, Breems D, van Putten WL, et al. Comparative analysis of the value of allogeneic hematopoietic stem-cell transplantation in acute myeloid leukemia with monosomal karyotype versus other cytogenetic risk categories. J Clin Oncol. 2012b;30:2140-6.

Couban S, Simpson DR, Barnett MJ, et al. A randomized multicenter comparison of bone marrow and peripheral blood in recipients of matched sibling allogeneic transplants for myeloid malignancies. Blood. 2002; 100:1525-31.

Craddock C, Nagra S, Peniket A, et al. Factors predicting long-term survival after T-cell depleted reduced intensity allogeneic stem cell transplantation for acute myeloid leukemia. Haematologica. 2010;95:989-95.

Craddock C, Labopin M, Pillai S, et al. Factors predicting outcome after unrelated donor stem cell transplantation in primary refractory acute myeloid leukaemia. Leukemia. 2011;25:808-13.

Craddock C, Jilani N, Siddique S, et al. Tolerability and clinical activity of post-transplantation azacitidine in patients allografted for acute myeloid leukemia treated on the RICAZA Trial. Biol Blood Marrow Transplant. 2016;22:385-90.

Craddock C, Hoelzer D, Komanduri KV. Current status and future clinical directions in the prevention and treatment of relapse following hematopoietic transplantation for acute myeloid and lymphoblastic leukemia. Bone Marrow Transplant. 2018. https://doi. org/10.1038/s41409-018-0203-8.

Cutler C, Logan B, Nakamura R, et al. Tacrolimus/sirolimus vs tacrolimus/methotrexate as GVHD prophylaxis after matched, related donor allogeneic HCT. Blood. 2014; 124:1372-7.

Devillier R, Harbi S, Furst S, et al. Poor outcome with nonmyeloablative conditioning regimen before cord blood transplantation for patients with high-risk acute myeloid leukemia compared with matched related or unrelated donor transplantation. Biol Blood Marrow Transplant. 2014;20:1560-5.

Dohner H, Estey EH, Amadori S, et al. Diagnosis and management of acute myeloid leukemia in adults: 
recommendations from an international expert panel, on behalf of the European LeukemiaNet. Blood. 2010;115:453-74.

Dohner H, Estey E, Grimwade D, et al. Diagnosis and management of AML in adults: 2017 ELN recommendations from an international expert panel. Blood. 2017;129:424-47.

Eapen M, Rocha V, Sanz G, et al. Effect of graft source on unrelated donor haemopoietic stem-cell transplantation in adults with acute leukaemia: a retrospective analysis. Lancet Oncol. 2010;11:653-60.

Eapen M, Logan BR, Horowitz MM, et al. Bone marrow or peripheral blood for reduced-intensity conditioning unrelated donor transplantation. J Clin Oncol. 2015;33:364-9.

Fasslrinner F, Schetelig J, Burchert A, et al. Long-term efficacy of reduced-intensity versus myeloablative conditioning before allogeneic haemopoietic cell transplantation in patients with acute myeloid leukaemia in first complete remission: retrospective followup of an open-label, randomised phase 3 trial. Lancet Haematol. 2018;5:e161-9.

Ferguson P, Hills RK, Grech A, et al. An operational definition of primary refractory acute myeloid leukemia allowing early identification of patients who may benefit from allogeneic stem cell transplantation. Haematologica. 2016;101:1351-8.

Gale RP, Horowitz MM, Rees JK, et al. Chemotherapy versus transplants for acute myelogenous leukemia in second remission. Leukemia. 1996;10:13-9.

Gondo H, Harada M, Miyamoto T, et al. Autologous peripheral blood stem cell transplantation for acute myelogenous leukemia. Bone Marrow Transplant. 1997;20:821-6.

Gooley TA, Chien JW, Pergam SA, et al. Reduced mortality after allogeneic hematopoietic-cell transplantation. N Engl J Med. 2010;363:2091-101.

Gorin NC, Labopin M, Frassoni F, et al. Identical outcome after autologous or allogeneic genoidentical hematopoietic stem-cell transplantation in first remission of acute myelocytic leukemia carrying inversion 16 or $\mathrm{t}(8 ; 21)$ : a retrospective study from the European Cooperative Group for Blood and Marrow Transplantation. J Clin Oncol. 2008;26: 3183-8.

Gratwohl A, Hermans J, Goldman JM, et al. Risk assessment for patients with chronic myeloid leukaemia before allogeneic blood or marrow transplantation. Chronic Leukemia Working Party of the European Group for Blood and Marrow Transplantation. Lancet. 1998;352:1087-92.

Gratwohl A, Stern M, Brand R, et al. Risk score for outcome after allogeneic hematopoietic stem cell transplantation: a retrospective analysis. Cancer. 2009; 115:4715-26.

Holter Chakrabarty JL, Rubinger M, Le-Rademacher J, et al. Autologous is superior to allogeneic hematopoietic cell transplantation for acute promyelocytic leukemia in second complete remission. Biol Blood Marrow Transplant. 2014;20:1021-5.
Jongen-Lavrencic M, Grob T, Hanekamp D, et al. Molecular minimal residual disease in acute myeloid leukemia. N Engl J Med. 2018;378:1189-99.

Koreth J, Schlenk R, Kopecky KJ, et al. Allogeneic stem cell transplantation for acute myeloid leukemia in first complete remission: systematic review and meta-analysis of prospective clinical trials. JAMA. 2009;301:2349-61.

Kroger N, Solano C, Bonifazi F. Antilymphocyte globulin for chronic graft-versus-host disease. N Engl J Med. 2016;374:1894-5.

Kroger N, Iacobelli S, Franke GN, et al. Dose-reduced versus standard conditioning followed by allogeneic stem-cell transplantation for patients with myelodysplastic syndrome: a prospective randomized phase III study of the EBMT (RICMAC Trial). J Clin Oncol. 2017;35:2157-64.

Lengfelder E, Lo-Coco F, Adès L, et al. Arsenic trioxidebased therapy of relapsed acute promyelocytic leukemia: registry results from the European LeukemiaNet. Leukemia. 2015;29:1084-91.

Lindsley RC, Saber W, Mar BG, et al. Prognostic mutations in myelodysplastic syndrome after stem-cell transplantation. N Engl J Med. 2017;376:536-47.

Lo-Coco F, Avvisati G, Vignetti M, et al. Retinoic acid and arsenic trioxide for acute promyelocytic leukemia. N Engl J Med. 2013;369:111-21.

Lowenberg B. Sense and nonsense of high-dose cytarabine for acute myeloid leukemia. Blood. 2013;121:26-8.

Lowenberg B, Ossenkoppele GJ, van Putten W, et al. High-dose daunorubicin in older patients with acute myeloid leukemia. N Engl J Med. 2009;361: 1235-48.

Martino R, de Wreede L, Fiocco M, et al. Comparison of conditioning regimens of various intensities for allogeneic hematopoietic SCT using HLA-identical sibling donors in AML and MDS with $<10 \%$ BM blasts: a report from EBMT. Bone Marrow Transplant. 2013;48:761-70.

Nagler A, Labopin M, Shimoni A, et al. Mobilized peripheral blood stem cells compared with bone marrow from HLA-identical siblings for reduced-intensity conditioning transplantation in acute myeloid leukemia in complete remission: a retrospective analysis from the Acute Leukemia Working Party of EBMT. Eur J Haematol. 2012;89:206-13.

Nash RA, Antin JH, Karanes C, et al. Phase 3 study comparing methotrexate and tacrolimus with methotrexate and cyclosporine for prophylaxis of acute graft-versus-host disease after marrow transplantation from unrelated donors. Blood. 2000;96:2062-8.

Oran B, Wagner JE, DeFor TE, Weisdorf DJ, Brunstein CG. Effect of conditioning regimen intensity on acute myeloid leukemia outcomes after umbilical cord blood transplantation. Biol Blood Marrow Transplant. 2011;17:1327-34.

Ossenkoppele GJ, Janssen JJ, van de Loosdrecht AA. Risk factors for relapse after allogeneic transplantation in acute myeloid leukemia. Haematologica. 2016;101:20-5. 
Parimon T, Au DH, Martin PJ, Chien JW. A risk score for mortality after allogeneic hematopoietic cell transplantation. Ann Intern Med. 2006;144:407-14.

Pasquini MC, Devine S, Mendizabal A, et al. Comparative outcomes of donor graft CD34+ selection and immune suppressive therapy as graft-versus-host disease prophylaxis for patients with acute myeloid leukemia in complete remission undergoing HLA-matched sibling allogeneic hematopoietic cell transplantation. J Clin Oncol. 2012;30:3194-201.

Quek L, Ferguson P, Metzner M, et al. Mutational analysis of disease relapse in patients allografted for acute myeloid leukemia. Blood Adv. 2016;1:193-204.

Robinson TM, O'Donnell PV, Fuchs EJ, Luznik L. Haploidentical bone marrow and stem cell transplantation: experience with post-transplantation cyclophosphamide. Semin Hematol. 2016;53:90-7.

Rollig C, Bornhauser M, Kramer M, et al. Allogeneic stem-cell transplantation in patients with NPM1mutated acute myeloid leukemia: results from a prospective donor versus no-donor analysis of patients after upfront HLA typing within the SAL-AML 2003 trial. J Clin Oncol. 2015;33:403-10.

Ruutu T, Gratwohl A, de Witte T, et al. Prophylaxis and treatment of GVHD: EBMT-ELN working group recommendations for a standardized practice. Bone Marrow Transplant. 2014;49:168-73.

Saad A, Lamb LS. Ex vivo T-cell depletion in allogeneic hematopoietic stem cell transplant: past, present and future. Bone Marrow Transplant. 2017;52:1241-8.

Sanz MA, Labopin M, Gorin NC, et al. Hematopoietic stem cell transplantation for adults with acute promyelocytic leukemia in the ATRA era: a survey of the European Cooperative Group for Blood and Marrow Transplantation. Bone Marrow Transplant. 2007;39:461-9.

Sanz MA, Grimwade D, Tallman MS, et al. Management of acute promyelocytic leukemia: recommendations from an expert panel on behalf of the European LeukemiaNet. Blood. 2009;113:1875-91.

Schlenk RF, Dohner K, Krauter J, et al. Mutations and treatment outcome in cytogenetically normal acute myeloid leukemia. N Engl J Med. 2008;358:1909-18.

Schlenk RF, Dohner K, Mack S, et al. Prospective evaluation of allogeneic hematopoietic stem-cell transplantation from matched related and matched unrelated donors in younger adults with high-risk acute myeloid leukemia: German-Austrian trial AMLHD98A. J Clin Oncol. 2010;28:4642-8.

Schmid C, Schleuning M, Schwerdtfeger R, et al. Longterm survival in refractory acute myeloid leukemia after sequential treatment with chemotherapy and reduced-intensity conditioning for allogeneic stem cell transplantation. Blood. 2006;108:1092-9.

Scott BL, Pasquini MC, Logan BR, et al. Myeloablative versus reduced-intensity hematopoietic cell transplantation for acute myeloid leukemia and myelodysplastic syndromes. J Clin Oncol. 2017;35:1154-61.

Sengsayadeth S, Savani BN, Blaise D, Malard F, Nagler A, Mohty M. Reduced intensity conditioning allo- geneic hematopoietic cell transplantation for adult acute myeloid leukemia in complete remission - a review from the Acute Leukemia Working Party of the EBMT. Haematologica. 2015;100:859-69.

Shouval R, Labopin M, Bondi O, et al. Prediction of allogeneic hematopoietic stem-cell transplantation mortality 100 days after transplantation using a machine learning algorithm: A European Group for Blood and Marrow Transplantation Acute Leukemia Working Party Retrospective Data Mining Study. J Clin Oncol. 2015;33:3144-51.

Soiffer RJ, Davids MS, Chen YB. Tyrosine kinase inhibitors and immune checkpoint blockade in allogeneic hematopoietic cell transplantation. Blood. 2018;131:1073-80.

Sorror ML, Maris MB, Storb R, et al. Hematopoietic cell transplantation (HCT)-specific comorbidity index: a new tool for risk assessment before allogeneic HCT. Blood. 2005;106:2912-9.

Sorror ML, Sandmaier BM, Storer BE, et al. Comorbidity and disease status based risk stratification of outcomes among patients with acute myeloid leukemia or myelodysplasia receiving allogeneic hematopoietic cell transplantation. J Clin Oncol. 2007;25:4246-54.

Sorror ML, Storb RF, Sandmaier BM, et al. Comorbidityage index: a clinical measure of biologic age before allogeneic hematopoietic cell transplantation. J Clin Oncol. 2014;32:3249-56.

Stelljes M, Krug U, Beelen DW, et al. Allogeneic transplantation versus chemotherapy as postremission therapy for acute myeloid leukemia: a prospective matched pairs analysis. J Clin Oncol. 2014;32:288-96.

Stone RM, Mandrekar SJ, Sanford BL, et al. Midostaurin plus chemotherapy for acute myeloid leukemia with a FLT3 mutation. N Engl J Med. 2017;377:454-64.

Tauro S, Craddock C, Peggs K, et al. Allogeneic stem-cell transplantation using a reduced-intensity conditioning regimen has the capacity to produce durable remissions and long-term disease-free survival in patients with high-risk acute myeloid leukemia and myelodysplasia. J Clin Oncol. 2005;23:9387-93.

Thomas X, Dombret H, Cordonnier C, et al. Treatment of relapsing acute promyelocytic leukemia by all-trans retinoic acid therapy followed by timed sequential chemotherapy and stem cell transplantation. APL Study Group. Acute promyelocytic leukemia. Leukemia. 2000;14:1006-13.

Todisco E, Ciceri F, Boschini C, et al. Factors predicting outcome after allogeneic transplant in refractory acute myeloid leukemia: a retrospective analysis of Gruppo Italiano Trapianto di Midollo Osseo (GITMO). Bone Marrow Transplant. 2017;52:955-61.

Versluis J, Labopin M, Niederwieser D, et al. Prediction of non-relapse mortality in recipients of reduced intensity conditioning allogeneic stem cell transplantation with AML in first complete remission. Leukemia. 2015;29:51-7.

Versluis J, Kalin B, Zeijlemaker W, et al. Graft-versusleukemia effect of allogeneic stem-cell transplantation and minimal residual disease in patients with 
acute myeloid leukemia in first complete remission. JCO Precis Oncol. 2017a. https://doi.org/10.1200/ PO.17.00078.

Versluis J, In't Hout FE, Devillier R, et al. Comparative value of post-remission treatment in cytogenetically normal AML subclassified by NPM1 and FLT3-ITD allelic ratio. Leukemia. 2017b;31:26-33.

Wagner JE Jr, Eapen M, Carter S, et al. One-unit versus two-unit cord-blood transplantation for hematologic cancers. N Engl J Med. 2014;371:1685-94.

Walter RB, Gooley TA, Wood BL, et al. Impact of pretransplantation minimal residual disease, as detected by multiparametric flow cytometry, on outcome of myeloablative hematopoietic cell transplantation for acute myeloid leukemia. J Clin Oncol. 2011;29: 1190-7.

Weisdorf D, Eapen M, Ruggeri A, et al. Alternative donor transplantation for older patients with acute myeloid leukemia in first complete remission: a center for international blood and marrow transplant researcheurocord analysis. Biol Blood Marrow Transplant. 2014;20:816-22.

Yanada M, Tsuzuki M, Fujita H, et al. Phase 2 study of arsenic trioxide followed by autologous hematopoietic cell transplantation for relapsed acute promyelocytic leukemia. Blood. 2013;121:3095-102.

Open Access This chapter is licensed under the terms of the Creative Commons Attribution 4.0 International License (http://creativecommons.org/licenses/by/4.0/), which permits use, sharing, adaptation, distribution and reproduction in any medium or format, as long as you give appropriate credit to the original author(s) and the source, provide a link to the Creative Commons license and indicate if changes were made.

The images or other third party material in this chapter are included in the chapter's Creative Commons license, unless indicated otherwise in a credit line to the material. If material is not included in the chapter's Creative Commons license and your intended use is not permitted by statutory regulation or exceeds the permitted use, you will need to obtain permission directly from the copyright holder. 